AperTO - Archivio Istituzionale Open Access dell'Università di Torino

\title{
Factor IX propeptide mutation and life threatening bleeding
}

\section{This is the author's manuscript}

Original Citation:

Availability:

This version is available http://hdl.handle.net/2318/93748

since 2018-09-04T14:29:42Z

Published version:

DOI:10.1016/j.thromres.2011.06.017

Terms of use:

Open Access

Anyone can freely access the full text of works made available as "Open Access". Works made available under a Creative Commons license can be used according to the terms and conditions of said license. Use of all other works requires consent of the right holder (author or publisher) if not exempted from copyright protection by the applicable law. 


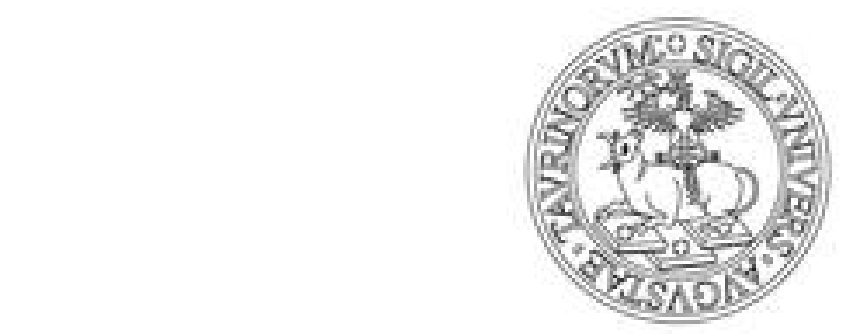

\section{UNIVERSITÀ DEGLI STUDI DI TORINO}

This Accepted Author Manuscript (AAM) is copyrighted and published by Elsevier. It is posted here by agreement between Elsevier and the University of Turin. Changes resulting from the publishing process - such as editing, corrections, structural formatting, and other quality control mechanisms - may not be reflected in this version of the text. The definitive version of the text was subsequently published in THROMBOSIS RESEARCH, 128, 2011, .

You may download, copy and otherwise use the AAM for non-commercial purposes provided that your license is limited by the following restrictions:

(1) You may use this AAM for non-commercial purposes only under the terms of the CC-BY-NC-ND license.

(2) The integrity of the work and identification of the author, copyright owner, and publisher must be preserved in any copy.

(3) You must attribute this AAM in the following format: Creative Commons BY-NC-ND license (http://creativecommons.org/licenses/by-nc-nd/4.0/deed.en), 


\title{
Factor IX propeptide mutation and life threatening bleeding
}

Conflict of interest statement The autor reported no potential conflicts of interest.

\author{
A. Vaccarino*, M. Bazzan, O. Giachino
}

Rare Disease, Immunological and Haematological Department University of Turin

*Corresponding author. Tel.: +390112402056 fax: +39 38012402052

E-mail address: antonella.vaccarino@libero.it (A. Vaccarino)

\section{P. Colagrande}

Internal Medicine Unit Torino Nord Emergenza San Giovanni Bosco Hospital

\section{P. Ferraresi}

Biochemistry and Molecular Biology University of Ferrara, Italy

\section{S. Stella D. Roccatello}

Rare Disease, Immunological and Haematological Department University of Turin

\section{F. Bernardi \\ Biochemistry and Molecular Biology University of Ferrara, Italy}

\section{Dear Editors,}

Bleeding events are the most relevant complications during oral anticoagulant treatment (OAT). They have been reported to occur at a rate of about 1.35 cases each 100 patients/year [1]. In very rare instances, OATrelated bleeding in patients with INR values within the therapeutic range may be associated with a mutation of the factor IX (FIX) gene. Two propeptide mutations have been described at locus- 10: Ala (GCC)-10 Val (GTC) and Ala (GCC)-10 Thr (ACC) [2,3]. The propeptide sequence at locus-10 plays an important role in the anticoagulant effect of vitamin $\mathrm{K}$ antagonists (VKA), since it contains the hepatic $\gamma$ carboxylase binding site [4]. In the presence of a propeptide locus-10 mutation, hepatic $\psi$-carboxylase binding to the FIX protein is markedly reduced. This reduced binding capacity has no clinical implications (except during OAT), since the FIX plasma level is within the normal range. When patients with the mutation are on OAT, the lack of biologically active vitamin $\mathrm{K}$ associated with reduced binding of $\mathrm{\gamma}$ carboxylase to the coagulation profactors, may induce a reversible but serious bleeding. This happens anytime, from days to weeks, after starting VKA treatment. The bleeding is caused by a rapid decrease in FIX activity (FIX:C), which drops to disproportionately lower values compared with the others vitamin-K dependent coagulation factors. FIX:C levels drop so low that laboratory data mimic acquired haemophilia B. Thus, patients with the mutation cannot be detected by routine monitoring of the INR. However, the sudden and disproportionately severe drop in factor IX plasma levels (b3\%) induced by OAT leads to persistently abnormal prolongation of activated partial thromboplastin time (aPTT). The morbidity associated with VKA-related bleeding, and the possibility of identifying mutated patients by performing aPTT has led us to question feasibility of aPTT screening in all patients on VKA. The main argumentation against aPTT screening is the low prevalence of the mutation [5-7]. A 61 year old man was admitted to the emergency department because of an "idiopathic" proximal deep-vein thrombosis of the right leg. He was treated with LMWH, followed by VKA. Heterozygosity for Factor V Leiden mutation, and no other 
thrombophilic abnormality, was found. After about two months of OAT the patient went to the emergency department of another hospital on account of severe pain and widespread ecchymosis of the right hand, which occurred the day after using a drill. Spontaneous muscle bleeding of the right thigh was also reported. Laboratory data showed an INR value of 2.15, but an abnormally prolonged aPTT ratio 4.93 (n.v. $\leq$ 1.2). The patient was discharged with a diagnosis of OAT related bleeding. He had never experienced any bleeding complications before starting OAT and there was no history of bleeding in his family. One month later, the patient returned to the same emergency department because of bilateral leg pain caused by deep muscle bleeding. INR was 2.1 and aPTT ratio was 5.63. The patient was again discharged with the same diagnosis. VKA dose was reduced and paracetamol was administered.

Two days later, the patient came to our hospital because of worsening of the bleeding, widespread ecchymosis on the chest, legs, hands and arms, and great difficulty in swallowing. Physical examination revealed tongue and pharynx haematoma. Laboratory data results were: haemoglobin $6.9 \mathrm{~g} / \mathrm{dl}$, INR 2.21 and aPTT ratio 5.38. VKA was discontinued and the patient underwent blood transfusion. Three days after discontinuing OAT, INR returned to 1.3 and aPTT ratio to 1.85 , while after seven days both INR and aPTT ratio returned to normal values (Fig. 1). At the same time, factors II, VII and X were assayed and found within normal range, while factor IX was about $40 \%$. Haematologic counselling was then requested. The patient's clinical history showed that both INR and aPTT ratio were within normal ranges before starting OAT. The patient reported no previous significant bleeding episodes, and familial bleeding tendency was excluded, while inherited bleeding tendency was unlikely. Due to the normal platelet count and normal antithrombin plasma levels, we ruled out disseminated intravascular coagulation. The possible presence of antibodies against factor VIII:C O IX:C was ruled out because of the quick and spontaneous return to normal aPTT ratio values after OAT discontinuation. The only anomaly we observed in coagulation tests was a disproportionate aPTT ratio prolongation when compared to INR prolongation. A literature search was then carried out and a mutation of the factor IX propeptide was hypothesized. This is a genetic predisposition to bleeding during OAT. Blood samples were sent off for molecular evaluation. The first three exons of the factor IX gene were amplified and sequenced. Whole exon 1 was amplified by the following primers: forward 5'-ATT GAG GGA GAT GGA CAT TAT T-3' (nt 2892-2913) and reverse 5'-AGT GAA GAA GAC AGC ATC AGA TAT-3' (nt 3159-3136); exons 2 and 3 were amplified together using forward 5'-TTC ATG ATG TTT TCT TTT TTG CTA-3' (nt 9237-9260) and reverse 5'-TGC AGA GAA AAA ACC CAC AT-3' (nt 9737-9718) primers (Gene Bank K02402.1). PCR amplifications were run for 30 cycles with a starting denaturation at 95 ${ }^{\circ} \mathrm{C}$ for $5 \mathrm{~min}, 95^{\circ} \mathrm{C}$ for $30 \mathrm{~s}, 58^{\circ} \mathrm{C}$ for $30 \mathrm{~s}$ and final extension at $72{ }^{\circ} \mathrm{C}$ for $7 \mathrm{~min}$. Direct nucleotide sequencing revealed a hemizygous -10Ala (GCC)-NThr (ACC) mutation. Only few case reports concerning the bleeding tendency in patients on OAT with factor IX propeptide mutation are present in the literature: most cases diagnosed in Switzerland and Germany were due to a founder mutation, with a clear regional focus [8-12]. To our knowledge this is the first case reported in Italy and actually it is not known if the mutation has an independent origin or results from a common founder. The bleeding tendency is usually severe, but the prevalence of the disorder in the general population is very low, although probably underdiagnosed. In our case, the patient had a very serious, life-threatening bleeding event. We highlight the fact that the significant aPTT prolongation was never considered a severe laboratory anomaly. Factor IX propeptide mutation is not well known among general practitioners or in the emergency setting. Due to the low prevalence of the defect in the general population, aPTT screening is proven not to be cost effective if evaluated in all patients on OAT. Data from literature and our experience suggest that if a male patient have significant bleeding events in the first weeks or months of OAT and INR is within the therapeutic range, the APTT should be performed. Immediate OAT withdrawal and replacement with an alternative anticoagulant treatment would prevent a worsening of bleeding symptoms. 


\section{References}

[1] Palareti G, Leali N, Coccheri S, Poggi M, Manotti C, D'Angelo A, et al. Bleeding complications of oral anticoagulant treatment: an inception-cohort, prospective collaborative study (ISCOAT). Lancet 1996;348:423-8.

[2] Chu K, Wu SM, Stanley T, Stafford DW, High KA. A mutation in the propeptide of Factor IX leads to warfarin sensitivity by a novel mechanism. J Clin Invest 1996;98: 1619-25.

[3] Oldenburg J, Quenzel EM, Harbrecht U, et al. Missense mutations at ALA-10 in the factor IX propeptide: an insignificant variant in normal life but a decisive cause of bleeding during oral anticoagulant therapy. $\mathrm{Br} \mathrm{J}$ Haematol 1997;98:240-4.

[4] Jorgensen MJ, Cantor AB, Furie BC, Brown CL, Shoemaker CB, Furie B. Recognition site directing vitamin K-dependent gamma-carboxylation resides on the propeptide of factor IX. Cell 1987;48:185-91.

[5] Van Der Meer FJ, Vos HL, Rosendaal FR. No indication for APTT screening in patients on oral anticoagulant therapy. Thromb Haemost 1999;81:364-6.

[6] Legnani C, Promezio M, Guazzaloca G, Coccheri S, Palareti G. Assessment of activated partial thromboplastin time and factor IX in subjects attending an anticoagulant clinic. Blood Coagul Fibrinolysis 2000;11:537-42.

[7] Ulrich S, Brand B, Oldenburg J, Asmis L. Congenital hypersensitivity to vitamin K antagonist due to FIX propeptide mutation at locus -10 : a (not so) rare cause of bleeding under oral anticoagulant therapy in Switzerland. Swiss Med Wkly 2008;138:100-7.

[8] Lammle B. Clinical problems with oral anticoagulation-3 case reports. Ther Umsch 2003;60:63-6.

[9] Oldemburg J, Kriz K, Wuillemin A, Maly FE, von Felten A, Siegemund A, et al. Genetic predisposition to bleeding during oral anticoagulant therapy: evidence for common founder mutations (FIXVal-10 and FIXThr10) and an independent CpG hotspot mutation (FIXthr-10). Thromb Haemost 2001;85:454-7.

[10] Bestmann L, Zuger M, Oldemburg J, Buhler D, Maly FE. Coagulation factor IX propeptide mutations causing coumarin hypersensitivity: identification of female alanine-10 valine hetrozygotes. Thromb Haemost 2001;85:567-8.

[11] Aegerter C, Fontana S, Fux C. Demarmels Biasiutti F. Life threatening bleeding under adequate oral anticoagulation. Cases 4a, b. Hamostaseologie 2003;23: 113-6.

[12] Jahns M. Massive muscle haematoma three months after starting vitamin $\mathrm{K}$ antagonist therapy for deep-vein thrombosis in an antithrombin deficient patient: another case of factor IX propeptide mutation. Thromb Haemost 2011;106. 
Fig. 1. International Normalized Ratio (INR-continuous line ) and Activated Partial Thromboplastin Time Ratio (aPTTR-dotted line) during Oral Anticoagulant Therapy (OAT). Arrows indicate bleeding events and OAT reduction or discontinuation.

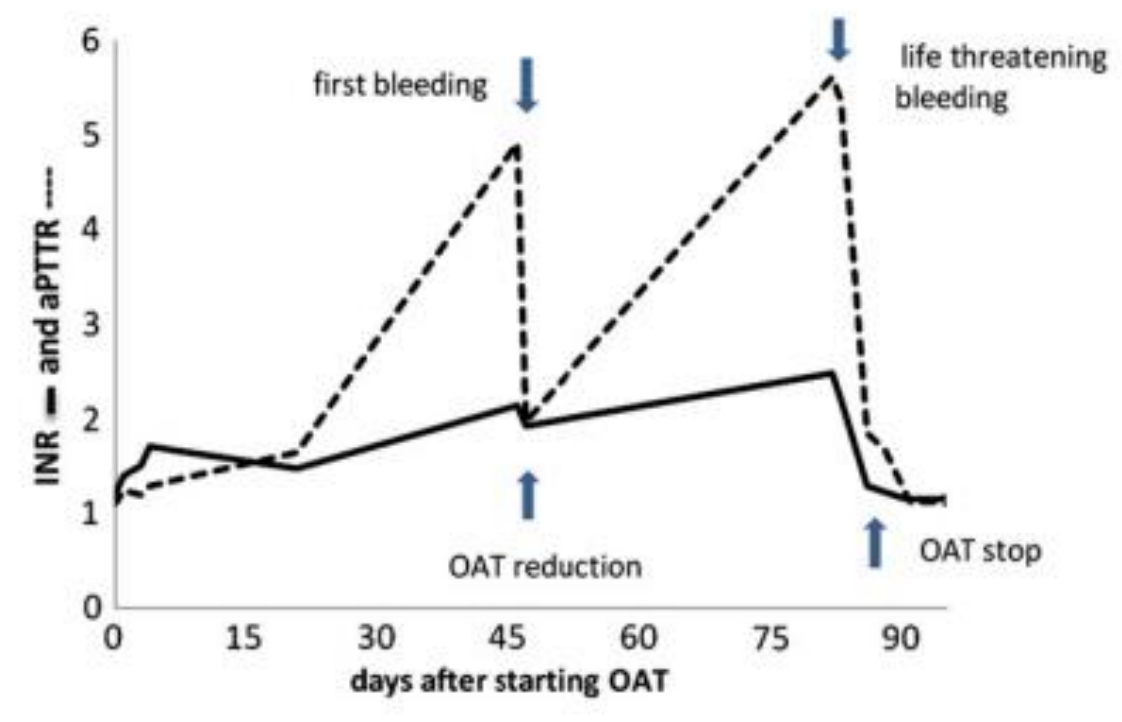

